\title{
MEJORAMIENTO DEL COMPORTAMIENTO SOCIALMENTE RESPONSABLE MEDIANTE INVESTIGACIÓN-ACCIÓN PARTICIPATIVA. UN ESTUDIO EN EL SURESTE DE MÉXICO
}

\author{
Improvement of socially responsible behavior through \\ participative action-research. A study in southeast Mexico
}

\section{Francisco Gerardo Barroso-Tanoira}

Recibido: 26 de Septiembre de 2021 - Aceptado: 18 de Octubre de 2021

\section{RESUMEN:}

Se presenta el diagnóstico general para evaluar el comportamiento socialmente responsable de una empresa mediana de refacciones automotrices, así como la intervención para mejorar dicho comportamiento. Los resultados muestran mejoría significativa en calidad de vida laboral, cuidado ambiental y colaboración con la comunidad, pero era necesario mejorar del liderazgo, el trabajo en equipo y la comunicación interna. También fueron evidentes el incremento de la productividad y la disminución de la rotación del personal.

\section{ABSTRACT}

The article reflects on different interdisciplinary postulates of territorial development immersed in the cultural, local sustainability and theoretical introspection towards a multifaceted institutionality and governance, which establishes strategies to generate windows-opportunity for a sustainable social-ecological resilience.

PALABRAS CLAVE: responsabilidad social empresarial, empresas medianas, investigaciónacción participativa, desarrollo organizacional, intervención en empresas de oportunidades. JEL: M14, L21

KEYWORDS: corporate social responsibility, middle sized enterprises, participative actionresearch, organizational development, intervention in enterprises.

JEL: M14, L21

${ }^{1}$ Profesor investigador. División de Negocios. Universidad Anáhuac Mayab. E-mail: francisco.barroso@anahuac.mx. Dirección: Km. 15.5 carretera Mérida-Progreso int. km. 2 carretera Chablekal. Mérida, Yucatán, México, C.P. 97310. Tel: (999)9424800 ext 516 


\section{Introducción}

\section{Planteamiento del problema y objetivos}

Como parte de sus actividades para mejoramiento constante, la empresa en estudio decidió participar en un programa de aceleración de negocios en la Oficina de Transferencia de una reconocida universidad privada en la ciudad de Mérida, Yucatán, en el sureste de México. Dicho programa incluye una sección en que se pide a cada empresa participante una evaluación de su comportamiento socialmente responsable, así como implementar mejoras en caso de detectarse áreas de oportunidad, lo cual dio origen a este estudio. De esta situación surgieron los objetivos de investigación, que son los siguientes: (1) verificar si el comportamiento de la empresa es socialmente responsable; (2) mejorar el comportamiento socialmente responsable de la empresa, y (3) determinar las causas que favorecerían una mejoría en dicho comportamiento.

\section{Justificación del estudio}

La experiencia relatada en esta investigación puede ser útil a otras empresas para verificar su propio comportamiento en cuanto a RSE y diseñar e implementar estrategias de manera que aprendan a detectar sus áreas de oportunidad y resolverlas por sí mismas. Por eso se utilizó la metodología de investigación-acción IA, en su modalidad de IAP, pues se trata de que cada empresa $u$ organización sea gestora de su propio aprendizaje.

Con este estudio se benefició una empresa en el sureste de México que, en cuanto a tamaño califica como mediana, según los criterios de la Secretaría de Economía (2009). Esta empresa de por sí ya era exitosa, pero con el mejoramiento en su comportamiento socialmente responsable también mejoró su desempeño general, haciendo evidente que ser socialmente responsable está al alcance de todos, además de que la metodología de IAP es adecuada para este tipo de estudios y para toda organización.

\section{Limitaciones}

La única limitación fue el tiempo de intervención, pues la empresa solo autorizó cuatro meses para no afectar sus ciclos estacionales de ventas. Y aunque los hallazgos son válidos únicamente para la empresa participante, por lo que no se pretende generalizarlos, la metodología sí puede ser replicada en otros contextos. Los resultados fueron recabados del 9 de julio al 8 de noviembre de 2018.

\section{Revisión de la literatura}

En esta sección se abordarán los conceptos generales de la responsabilidad social empresarial (RSE), así como los lineamientos de la investigación-acción (IA) e investigaciónacción participativa (IAP).

\section{Concepto de responsabilidad social empre- sarial (RSE)}

Puede definirse la responsabilidad social empresarial (RSE) como la contribución activa y voluntaria de las empresas hacia el mejoramiento social, económico y ambiental (Libro Verde de la Unión Europea, 2001, citado por Porto y Castromán, 2006). Se pretende ir más allá del mero cumplimiento de las leyes (Pérez-Espinoza et al., 2016) creando riqueza de manera justa, lo que significa ser rentable respetando la dignidad y los derechos inalienables de las personas (Fernández-García, 2018). El enfoque al bien común debe llevarlas a crear riqueza de manera justa y eficiente, respetando la dignidad y los derechos inalienables de los individuos (Ramos, 2006), lo cual implica evitar actos de corrupción en el gobierno corporativo y en las operaciones de toda la organización, así como implementar sanciones si ocurrieran. Esto significa cumplir con las expectativas de todos sus participantes o stakeholders en lo particular, y de la sociedad en lo general, respetando la dignidad de la persona y de las comunidades en que opera, así como su entorno.

El Centro Mexicano para la Filantropía (CE$\mathrm{MEFI}$ ) incluye cuatro ámbitos que debe cumplir toda empresa socialmente responsable y mediante los cuales las califica para otorgarles el distintivo de Empresa Socialmente Responsable [ESR] (Porto y Castromán, 2006; Barroso-Tanoira, 2008):

1) Contribuir a la calidad de vida dentro de la empresa.

2) Cuidado y preservación del medio ambiente.

3) Desempeñarse con un código de ética. 
4) Vincularse con la comunidad a partir de la misión del negocio, pero también de los bienes y servicios producidos

Las empresas socialmente responsables, en el sentido amplio del concepto, deben cumplir con los cuatro ámbitos al mismo tiempo (Barroso-Tanoira, 2008). Si practican algunos de ellos entonces sólo estarán realizando acciones de compromiso empresarial con la sociedad, pero no puede decirse que sean socialmente responsables en el sentido completo del concepto.

\section{Investigación-acción}

Álvarez-Gayou (2003) indica que el propósito de la investigación-acción (IA) es resolver problemas cotidianos e inmediatos tratando de hacer comprensible el mundo social y buscando la mejora de la calidad de vida de las personas. Elliott (2000) la define como el estudio de una situación social con miras a mejorar la calidad de la acción dentro de ella. La organización o unidad de análisis toma las decisiones con la ayuda del investigador, pero no bajo el mando del investigador, pues se pretende que la organización sea gestora de su propio aprendizaje (Colmenares, 2012) y que en el futuro pueda continuar el proceso por su cuenta. No se buscan respuestas correctas o incorrectas como lo plantean los métodos positivistas, sino soluciones basadas sobre los puntos de vista e interpretaciones de aquellos involucrados en la investigación, por lo que se emplean estrategias cualitativas.

Para Bernal (2010), el proceso metodológico de IA consta de tres fases: (1) fase inicial o de contacto con la comunidad; (2) fase intermedia o de elaboración de un plan de acción, y (3) fase de ejecución y evaluación del estudio.

\section{Los ciclos de intervención.}

McKernan, citado por Álvarez-Gayou (2003), planteó un método de proceso de IA que se desarrolla mediante ciclos de intervención, llamados también ciclos de acción reflexiva o propuestas de cambio. Se basa en lo siguiente: 1. Los participantes que están viviendo un problema son los mejor capacitados para abordarlo en un entorno naturalista, conside- rando al sujeto como poseedor de capacidad de acción y poder transformados, lo que coincide con Arellano, citado por Bernal (2010).

2. La conducta de estas personas está influida de manera importante por su entorno natural. Se busca la transformación de la realidad de dicha comunidad (grupo o colectivo). 3. La metodología cualitativa es la más conveniente para el estudio de los entornos naturalistas, puesto que es uno de sus pilares epistemológicos.

Después de un diagnóstico inicial para detectar las áreas generales de oportunidad, el primer ciclo de acción consiste en los intentos por definir lo más claramente posible la situación o el problema, para luego pasar a la detección y evaluación de las necesidades a abordar, estableciéndose las limitaciones internas y externas del proceso. Se busca que surjan ideas, propuestas e hipótesis, las cuales se asumen como ideas inteligentes y no como soluciones. Luego se analiza el plan general de acción, el cual se lleva a la práctica y se evalúa. En esta evaluación los participantes buscan comprender los efectos y lo que han aprendido.

En el segundo ciclo y en los sucesivos se produce una nueva definición del problema para realizar otra evaluación de las necesidades, a partir de las cuales surgen más ideas o hipótesis que llevan a la revisión del plan anterior y a la elaboración de uno para cada ciclo.

Para Rojas, citado por Bernal (2010), la participación de la gente en un proceso de IA no es sólo una posibilidad concedida a la comunidad (entendida como todo grupo sujeto de investigación), sino un derecho que tiene toda persona para ser sujeto y protagonista de su propio proyecto de vida.

\section{Investigación-acción participativa.}

En la investigación acción-acción participativa (IAP), el investigador se identifica con los grupos con que desarrolla la investigación (Colmenares, 2012) y contribuye al cambio social en unión con los grupos mismos, por lo que ambos pueden ser sujetos y objetos de estudio (Bernal, 2010; De-Oliveira-Figueiredo, 2015). La IAP toma técnicas de diversas 

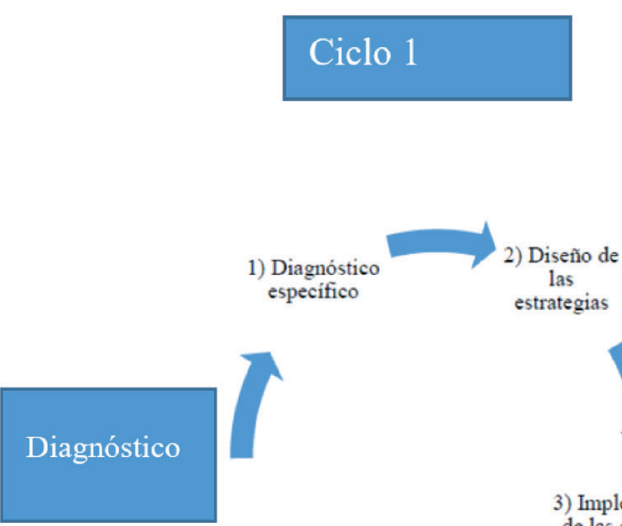

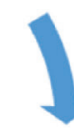

3) Implementación de las estrategias

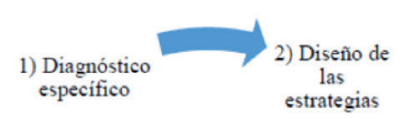

las
estrategias

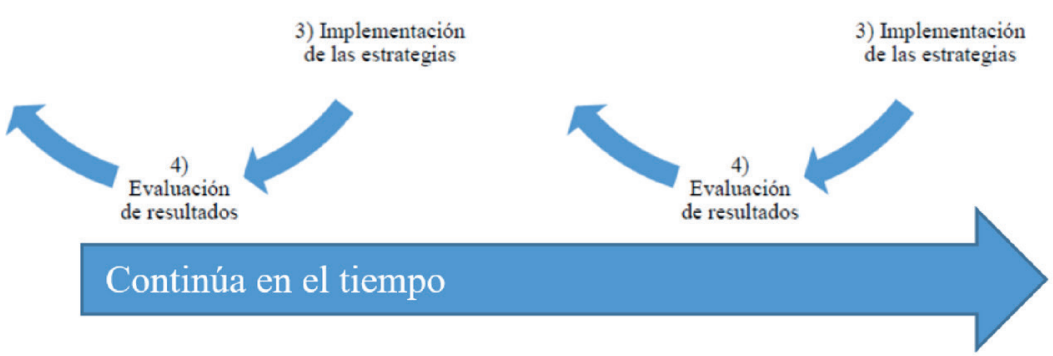

Figura 1: Ciclos de investigación-acción. Elaboración propia con base en McKernan, citado por Álvarez-Gayou (2003).

disciplinas, que van desde encuestas hasta la revisión de documentos, administración de entrevistas, formación de grupos motivacionales, reportes de trabajo de campo, historias de vida y el uso de la crónica (relato), según sea el escenario de investigación y los objetivos del proyecto de intervención (Sirvent, 2008).

\section{Metodología}

El estudio consta de dos fases: (1) diagnóstico, en el que se identificaron las acciones de RSE que lleva a cabo la empresa para descubrir aquello susceptible de mejora, y (2) una intervención para mejorar el comportamiento socialmente responsable de la empresa participante, basada en IAP.

\section{Fase 1: diagnóstico}

\section{Alcance y diseño.}

El diagnóstico fue de alcance exploratorio y luego descriptivo, con diseño no experimental transversal debido a que no hubo manipulación deliberada de las variables (Hernández-Sampieri y Mendoza, 2018), ya que el investigador se limitó solamente a registrar los resultados e interpretarlos. El enfoque fue cuantitativo y el método fue deductivo con trabajo de cam- po, recurriendo a la encuesta como técnica y al cuestionario como instrumento.

\section{Contexto y participantes.}

La empresa en estudio comenzó a operar el 7 de abril del año 2000 en la ciudad de Mérida, Yucatán, en el sureste de México. Sus fundadores estaban conscientes de la necesidad de otorgar mejores servicios para el mercado de refacciones automotrices, buscando la satisfacción de sus clientes con una cultura basada en calidad, honestidad, servicio y buen precio. Su misión es "satisfacer las necesidades del mercado automotriz con servicios y productos de calidad, contribuyendo al éxito de sus clientes con refacciones de calidad, servicios y atención profesional".

Ampliamente reconocida en el mercado, está afiliada a las principales asociaciones empresariales de la región. Cabe mencionar que en el momento del estudio contaba con nueve sucursales ubicadas estratégicamente dentro de la ciudad y se ha consolidado como una de las más importantes en su ramo. Cuenta con un extenso surtido de refacciones para todo tipo de automóvil y camión tanto de manufactura nacional como 
de importación (AGP, 2018).

Participaron todos los trabajadores de la empresa (son 95), de los cuales uno era el Director General, 18 gerentes y 76 trabajadores de las nueve tiendas y oficinas. Al ser un estudio general se dio el mismo peso a todas las respuestas sin distinguir entre jefes y trabajadores. La edad promedio de los participantes era de 33 años y la antigüedad promedio de 31 . En cuanto a sexo, $52 \%$ son mujeres.

\section{Instrumentos y procedimiento.}

Se utilizó un cuestionario diseñado y validado por el autor de este trabajo (ver la Figura 3), basado en los cuatro ámbitos propuestos por el CEMEFI (Barroso-Tanoira, 2008). Fue administrado por el personal designado por la empresa durante abril de 2018. Para verificar la confiabilidad se calculó el coeficiente alfa de Cronbach comprobatorio, el cual resultó de 0,94, lo que ratificó la estabilidad del instrumento. También se realizó un análisis factorial comprobatorio por el método de componentes principales y rotación varimax para hallar las varianzas explicadas correspondientes a los factores más críticos y que necesitan atención, los cuales pueden observarse en la Figura 4.

\begin{tabular}{|c|c|c|}
\hline Escala de puntos & Interpretación del resultado según escala & Clave \\
\hline 1 a 1.49 & Totalmente en desacuerdo & \\
\hline 1.5 a 2.49 & En desacuerdo & \\
\hline 2.5 a 3.49 & Más o menos de acuerdo & \\
\hline 3.5 a 4.49 & De acuerdo & \\
\hline 4.5 a 5 & Totalmente de acuerdo & \\
\hline
\end{tabular}

Figura 2: Escala para interpretación de los datos. Elaboración propia.

Para el análisis de los resultados se recurrió a las utilerías de Excel, así como al programa estadístico SPSS. Para interpretarlos, se utilizó la escala que se observa en la Figura 2. Para la información cualitativa proveniente de las preguntas abiertas al final del cuestionario, las respuestas se agruparon por frecuencia de mención en ideas clave, que a su vez fueron agrupadas en categorías definidas (Álvarez-Gayou, 2003). Cabe mencionar que se preguntó a la empresa la tasa de rotación del personal, la cual se ubicó en un promedio de 5.5\% mensual, así como la productividad, medida como el porcentaje de alcance de las metas programadas para cada individuo, la cual se ubicaba en $74 \%$ en promedio.

\section{Resultados}

A continuación, se presentan los resultados generales del diagnóstico. (Ver fig. 3)

\section{Respuesta a preguntas abiertas.}

Los comentarios recabados en la sección de preguntas abiertas son los siguientes, agru- pados en categorías por frecuencia de mención (de más mencionado a menos):

\section{Horario laboral.}

- Las horas de trabajo son muchas, por lo que hay poco tiempo para la familia. Si se permitiera a los trabajadores estar más tiempo con sus familias, estarían más plenos y contentos en el trabajo (seis menciones)

- El ambiente de trabajo es bueno. Sin embargo, el horario es muy largo.

- Deberían ser un poco más considerados con las situaciones que puedan ocasionar un retraso en la entrada del empleado, siempre y cuando dicho retraso esté justificado.

- Que se implementen turnos de guardia los sábados

\section{Comunicación y liderazgo.}

- Los trabajadores son más participativos y expresan sus puntos de vista

- Que realmente se escuche al trabajador sin tener consecuencias

- Los trabajadores creen que cualquier error 


\begin{tabular}{|c|c|c|c|c|}
\hline & Parte 1: calidad de vida en la empresa & Promedio & Resultado & $\begin{array}{l}\text { De sul acien } \\
\text { esta nctar }\end{array}$ \\
\hline 1 & La empresa apoya nuestro desarrollo & 3,9 & De acuerdo & 0,80 \\
\hline 2 & La empresa ncs capacita & 3,9 & De acuerdo & 1,03 \\
\hline 3 & Los sualdos que paga la empresa son competitivos en el merca do la boral & 3,5 & De acuerdo & 0,94 \\
\hline 4 & Contamos con prestaciones competitivas & 3,5 & De acuerdo & 1,00 \\
\hline 5 & Puedo combinar mi trabajo con mi vida personal & 3,0 & Más o menos de acuerdo & 1,03 \\
\hline 6 & Las condiciones de trabajo son adecuadas & 3,8 & De acuerdo & 0,85 \\
\hline 7 & Conczco la visión de la empresa & 3,9 & De acuerdo & 0,87 \\
\hline 8 & Conozco la misión de la empresa & 3,8 & De acuerdo & 0,97 \\
\hline 9 & E liderazgo en la empresa es adecuado & 3,7 & De acuerdo & 0,94 \\
\hline & Se respeta nuestra dignidad como personas & 4,0 & De acuerdo & 0,77 \\
\hline & Promedio & 3,7 & De acuerdo & \\
\hline
\end{tabular}

\begin{tabular}{|c|c|c|c|c|}
\hline & Parte 2: aidado y preservación del medio ambiente & Promedio & Resultado & $\begin{array}{l}\text { De sulacien } \\
\text { esta nctar }\end{array}$ \\
\hline 14. & La empresa se preocupa por el cuidado del medio ambiente & 3,7 & De acuerdo & 0,65 \\
\hline 15 & La empresa fomenta en sus trabaj adores el cuidado del medio ambiente & 3,3 & Más o menos de acuerdo & 1,23 \\
\hline 16 & La empresa participa en actividades que cuiden el medio ambiente & 28 & Más o menos de acuerdo & 1,33 \\
\hline 17 & Se capacita al personal para el aidado del ambiente & 2,5 & Más o menos de acuerdo & 1,22 \\
\hline 18 & La empresa organiza actividades para el cuidado ambiental & 2,6 & Más o menos de acuerdo & 1,38 \\
\hline 19 & La empresa cola bora con organismos o instituciones dedicadas al cuidado am biental & 2,6 & Más o menos de acuerdo & 1,42 \\
\hline 20 & Se respeta el entorno ecol ógicoen sus procesos de operación. & 3,3 & Más o menos de acuerdo & 1,09 \\
\hline & Promedio & 3,0 & Más o menos de acuerdo & \\
\hline
\end{tabular}

\begin{tabular}{|c|c|c|c|c|}
\hline & Parte 3 a: trato ético hadia los involucrados con la empresa (stakeholders) & Promedio & Resultado & $\begin{array}{l}\text { De sutacion } \\
\text { estancar }\end{array}$ \\
\hline 21 & E trato de la empresa es justo hacia los que laboran en ella & 3,4 & Más o menos de acuerdo & 1,04 \\
\hline 22 & Subir de puesto en la empresadepende del esfuerzo de cada trabajador & 42 & De acuerdo & 0,92 \\
\hline 23 & La comunicación en la empresa prom ueve un buen clima laboral & 3,6 & De acuerdo & 1,09 \\
\hline 24 & Se toma en cuenta al personal en las decisiones de la empresa & 3,3 & Más o menos de acuerdo & 1,44 \\
\hline 25 & Se toma en cuenta a los externos (accionistas, proveedores, clientes) en las decisiones de la empresa & 4,0 & De acuerdo & 0,94 \\
\hline 25 & E trato de la empresa hacia los externos (accionistas, proveedores, clientes) es justo & 3,6 & De acuerdo & 1,19 \\
\hline & Promedio & 3,7 & De acuerdo & \\
\hline
\end{tabular}

\begin{tabular}{|c|c|c|c|c|}
\hline & Parte 4 a: rel ación con la comunidad & Promedio & Resultado & $\begin{array}{l}\text { De suladion } \\
\text { esta ndar }\end{array}$ \\
\hline 27 & La empresa ayuda a movimientos de caridad (fil antrop'a) & 3,1 & Más o menos de acuerdo & 1,74 \\
\hline 29 & La empresa promueve hada la comunidad una cultura de responsabilidad social & 3,4 & Más o menos de acuerdo & 1,52 \\
\hline 30 & La empresa se desempeĩa con un código de ética & 3,3 & Máso menos de acuerdo & 1,18 \\
\hline & Promedio & 3,3 & Más o menos de acuerdo & \\
\hline
\end{tabular}

Figura 3: Resultados del cuestionario de diagnóstico

puede causar su baja de la empresa

- Falta comunicar de mejor manera los logros de la empresa

- Que los trabajadores conozcan lo que hacen otras áreas de la empresa

- Falta de liderazgo adecuado

- Hay ideas muy obsoletas y que deben cambiar

\section{Calidad de vida.}

- Ha habido cambios en el compromiso con la empresa y la responsabilidad de trabajar, siempre buscando el bien de la familia.

- Es necesario buscar mejor calidad de vida de los trabajadores con políticas que sean costeables

\section{Acciones de ayuda social.}

- Se recolecta cartón y PET para beneficio de los trabajadores

- Se han recolectado víveres para personas damnificadas en el terremoto de 2017 en la Ciudad de México

\section{Equidad.}

- El trato es justo e igualitario para todo el personal

- Se requiere que se reconozca el trabajo de cada uno

- Los jefes de departamentos delegan funciones, pero no hablan de incremento de sueldo - Se necesitan bonos reales de productividad para incentivar al empleado 


\section{Capacitación.}

- La empresa se preocupa por el desarrollo del personal capacitándolo constantemente

- Debe de haber capacitación más clara y concisa

Como puede verse, la mayoría de los comentarios se referían al horario laboral, especialmente a las extensas horas de trabajo. Faltaban comunicación, reconocimiento e incentivos. $Y$ aunque se realizaban acciones sociales, faltaba comunicarlas al personal de la empresa.

\section{Resultado del análisis factorial.}

Los resultados cargaron en cinco factores, los cuales resultaron críticos, representando el $90.96 \%$ de la varianza explicada, por lo que requerían atenderse prioritariamente, pues una variación en ellos afectaría de manera significativa los esfuerzos de RSE (ver Figura 4).

\begin{tabular}{|c|c|c|c|}
\hline Variable redefinida & Promedio & Significado & $\begin{array}{c}\text { Varianza } \\
\text { explicada } \\
(\%)\end{array}$ \\
\hline Preservación del ambiente & 3.1 & Más o menos de acuerdo & 27.57 \\
\hline Comunicación y desarrollo personal & 3.7 & De acuerdo & 22.05 \\
\hline Liderazgo y ética empresarial & 3.5 & De acuerdo & 20.87 \\
\hline Remuneración y horario laboral & 3.3 & Más o menos de acuerdo & 11.27 \\
\hline Conocimiento de la misión y visión & 3.9 & De acuerdo & 9.20 \\
\hline
\end{tabular}

Figura 2: Factores redefinidos significativos con sus respectivas varianzas. Para la interpretación de los promedios, se consideró la escala de la figura 2

\section{Conclusiones del diagnóstico.}

La empresa practicaba un comportamiento socialmente responsable en el sentido amplio del concepto, como indica BarrosoTanoira (2008), debido a que realiza acciones en los cuatro ámbitos según el CEMEFI. Todos los participantes estuvieron de acuerdo en que la calidad de vida era adecuada, así como con el trato ético que les ofrecía. Sin embargo, era necesario que en la empresa se hicieran acciones para preservación del medio ambiente, para la vinculación con la comunidad y para que los trabajadores compaginaran su vida con el trabajo. Los factores críticos fueron (ver la Figura 4): (1) Preservación del medio; (2) Comunicación y desarrollo personal; (3) Liderazgo y ética empresarial; (4) Remuneración y horario laboral, y (5) Conocimiento de la visión y misión de la empresa.

Un punto importante fue que cerca del 15\% de los trabajadores no sabía si la empresa se involucraba en acciones de RSE, ni de filantropía o de ayuda social, lo que reforzó la necesidad de mejorar la comunicación interna.
Entre los factores de calidad de vida se encontraban la necesidad de reconocimiento, desarrollo personal y capacitación efectiva.

\section{Recomendaciones para la intervención.}

Era necesario que la intervención se realizara para mejorar los puntajes de aquellos ámbitos en que estuvieron bajos, para lo que se recomendó a la empresa:

1) Comenzar con educación para la preservación del medio ambiente, así como acciones concretas que los trabajadores pudieran realizar para este efecto.

2) Instaurar un medio de comunicación interno (gaceta, periódico mural, revista) para que los trabajadores estuvieran al tanto de lo que se hace en la empresa. Se recomendó incluir información sobre los cumpleaños, cambios de puesto y logros del personal.

3) Aunque la mayoría de los trabajadores conoce la misión, sería adecuado tener reuniones periódicas con ellos para reflexionar sobre los ejes fuerza de la misma y trazar actividades para el mejoramiento del desempeño empresarial.

4) Diseñar alguna actividad o implementar 
alguna iniciativa para vinculación con la comunidad.

5) Fomentar el liderazgo, el trabajo en equipo y la toma de decisiones en la empresa, según la naturaleza del puesto y trabajo.

6) Verificar qué podía hacerse para que el trabajador compaginara su vida con el trabajo, de manera que mejore la calidad de vida laboral.

\section{Fase 2: intervención}

\section{Tipo y alcance.}

En esta fase el estudio fue descriptivo evolucionando a explicativo (Hernández-Sampieri y Mendoza, 2018), constituyendo un estudio de caso utilizando la metodología de IAP debido a que se pretendía estudiar el proceso de cambio social desde la empresa misma, de manera que ésta fuera gestora de su propio aprendizaje. Esto indica que el enfoque fue cualitativo, aunque se utilizaron herramientas cuantitativas para verificar la eficacia de la intervención mediante una pre prueba y post prueba.

\section{Participantes.}

Participaron los 95 trabajadores de la empresa. Para representarlos se constituyó un equipo de investigación-acción participativa (IAP), liderado por el Director General, y del cual el autor de este trabajo formó parte. Participaron además la Gerente de Recursos Humanos, la Directora Administrativa, el Director Comercial, el Director de Recursos Físicos e Inventario, el Director de Sistemas y la Gerente de Compras. Ocho personas en total, siete de ellas representantes de los diversos sectores de la empresa, de manera que pudiera asegurarse la participación de todos en la construcción de nuevo conocimiento, tal como sugieren Bernal (2010) y Colmenares (2012). En la intervención se involucró a todo el personal de la empresa, que fueron los 95 que participaron en el diagnóstico inicial.

\section{Instrumentos.}

El formato utilizado en el diagnóstico (ver la Figura 3) se tomó como pre-prueba, por lo que también se aplicó a todo el personal al término del segundo ciclo de intervención para verificar si hubo alguna mejoría en cuanto a la percepción del comportamiento socialmente responsable de la empresa. La información cuantitativa fue analizada mediante las utilerías de Excel, recurriendo al SPSS para verificación de diferencias de medias. Las respuestas cualitativas se agruparon por frecuencia de mención en ideas clave y éstas, a su vez, en categorías definidas (Álvarez-Gayou, 2003).

\section{Procedimiento de la intervención.}

El desarrollo de la metodología a través de los ciclos de intervención es la que se muestran para la IA (ver la Figura 1). Y como se comentó anteriormente, la intervención duró del 9 de julio al 8 de noviembre de 2018, que es el tiempo que autorizó la empresa para no afectar sus actividades ni las ventas estacionales de fin de año. Dicho período se dividió en dos ciclos o propuestas de cambio, de dos meses cada uno: el primero, del 9 de julio al 8 de septiembre, y el otro del 9 de septiembre al 8 de noviembre. Aunque habría sido deseable que cada ciclo fuera más largo, las condiciones de la empresa lo impusieron así. Sin embargo, fue acordado por el Comité de IAP que éste sería un tiempo razonable como proyecto piloto, por lo que el proceso continuaría en el futuro por parte de la propia empresa. Las reuniones del Comité IAP fueron semanales y se levantó un acta, a manera de memoria, de cada reunión, registrando avances, revisiones y acuerdos para la siguiente reunión.

\section{Ciclo 1. Primera propuesta de cambio.}

De los resultados y recomendaciones del diagnóstico general, el Comité IAP decidió atender las siguientes áreas de oportunidad: (1) Mejorar la comunicación interna; (2) Mejoramiento del medio ambiente; (3) Mejorar la relación con la comunidad, y (4) Mejorar la calidad de vida en la empresa, planteando los objetivos y estrategias que se presentan en el esquema de la Figura 1.

Para mejorar la comunicación interna, que es el Área de Oportunidad 1 y la primera abordada, se acordó implementar un medio interno de comunicación, el cual consistió en retomar la gaceta informativa de la empresa y distribuirla tanto en papel como electróni- 
camente en la página Web una vez al mes. Esto se implementó desde el primer mes del ciclo. Se intentó mejorar la comunicación interna a través de tecnologías de la información (TICS), redes sociales y un buzón de sugerencias, pero no se logró. Lo que sí se hizo fue retomar las reuniones quincenales del Comité Empresarial de la empresa, integrado por los directivos y gerentes, en el que se discuten aspectos de administración, contrataciones, capacitación, ventas, mercadotecnia y se toman decisiones estratégicas.

Se diseñaron estrategias para el mejoramiento del medio ambiente, que corresponde al Área de Oportunidad 2, las cuales consistieron en acciones de reciclaje, separación de basura y reforestación. Solo se pudieron implementar las acciones de reciclaje organizando los botes de basura y programando los días de recolección, nombrando un encargado por cada tienda.

Para el Área de Oportunidad 3 se implementó un plan de limpieza del sitio en donde cada sucursal opera, con el objetivo de contribuir a mejorar la imagen de cada lugar y la relación con los vecinos. Cabe mencionar que cuando comenzaron a hacerlo, las empresas y residentes de la misma calle comenzaron a imitarlos. Esto comprueba que el ejemplo es la mejor forma de inspirar las acciones de otros.

En cuanto al Área de Oportunidad 4, que es mejorar la calidad de vida en la empresa, se contactó a dos universidades y a un centro médico público para las pruebas de salud de los trabajadores. No se logró implementar las guardias los sábados ni reorganizar cargas laborales y tampoco se logró difundir la misión y visión entre los trabajadores, de manera que pudieran conocerlas, identificarlas y vivirlas.

Como puede verse, se cumplieron cinco actividades y se comenzó una, resultando que en promedio su avance fue de $38 \%$. Se acordó que los puntos que quedaran pendientes o iniciados se abordaran en el siguiente ciclo (ver la Figura 5).

\section{Ciclo 2. Segunda propuesta de cambio.}

El Comité IAP decidió no iniciar nuevas acciones concretas, sino continuar con las que ya se estaban haciendo, asegurándose de dar a los responsables todo el apoyo necesario. Para el Área de Oportunidad 1, ahora sí se implementó una estrategia comunicación interna a través de redes sociales. Se añadieron los logros de la empresa en la página Web, además de la gaceta (ya implementada desde el ciclo anterior), de manera que todos en la empresa estuvieran enterados de los acontecimientos. Se diseñó la mecánica del buzón de sugerencias, pero no se implementó en este ciclo.

En cuanto al Área de Oportunidad 2, se organizaron acciones para separar la basura y se puso en marcha el plan de reforestación. También se logró el vínculo con una institución ambientalista (denominada Kanan $K a b)$, la que impartió las sesiones sobre conservación del medio ambiente. Quedó una valoración final del conocimiento adquirido para enero de 2019, la cual se realizó según lo planeado.

Las acciones del Área de Oportunidad 3 continuaron y para el Área de Oportunidad 4 finalmente se implementaron guardias los sábados y se readecuaron las cargas laborales, pero solo hubo un avance del $50 \%$ en cuanto a las actividades para difundir la misión y visión. Quedó pendiente para un siguiente ciclo el diseño e implementación de estrategias para promover el deporte entre los integrantes de la empresa. Como puede verse en la Figura 5, el avance fue del $81 \%$ en general con respecto al inicio del Ciclo 1, lo cual motivó mucho al Comité IAP para seguir adelante con los procesos de cambio.

\section{Post prueba.}

Al término del Ciclo 2, con lo que termina la intervención para efectos de este estudio, se administró a todos los trabajadores de la empresa el mismo cuestionario que en el diagnóstico, de manera que se pudiera ver si se dio alguna mejoría en cuanto al comportamiento organizacional según los ámbitos de la RSE. (ver la Figura 6). 


\begin{tabular}{|c|c|c|c|c|c|c|c|}
\hline \multicolumn{8}{|c|}{ INTERVENCIÓN } \\
\hline ENPRESA: & Refaccionaria & & & & & & \\
\hline $\begin{array}{l}\text { Área de oportunidad } \\
\text { (Eje) }\end{array}$ & Objefvo a lograr & Acciones concretas & Indica dor & $\begin{array}{c}\text { Resul ta dos (al } \\
\text { final del Ciclo 1) }\end{array}$ & $\begin{array}{c}\text { Resulta dos } \\
\text { en cifras } \\
\text { (Ciclo 1) }\end{array}$ & $\begin{array}{c}\text { Resultados (al final } \\
\text { del Ciclo 2) }\end{array}$ & $\begin{array}{l}\text { Resultados } \\
\text { en cifras } \\
\text { (Ciclo 2) }\end{array}$ \\
\hline \multirow{5}{*}{$\begin{array}{l}\text { 1) Mejorar la } \\
\text { comunicacón interna }\end{array}$} & \multirow{5}{*}{$\begin{array}{l}\text { 1.1. Implementar un } \\
\text { medio inteno de } \\
\text { comunicacón }\end{array}$} & $\begin{array}{c}\text { Retomar la gaceta } \\
\text { informativa "Todo AGP" y } \\
\text { publica rla mesua mente }\end{array}$ & $\begin{array}{c}\text { Elaboración de } \\
\text { la gaceta } \\
\text { mensual (cada } \\
1 \text { de mes) }\end{array}$ & $\begin{array}{l}\text { Se cumplió } \\
\text { totalmente }\end{array}$ & 100 & $\begin{array}{c}\text { Se siguió con un } \\
\text { formato diferente. Hay } \\
\text { que mejorar en cuanto } \\
\text { a envia to } \\
\text { electrónicamente. } \\
\text { Proyecto de imprimir la } \\
\text { gaceta. Lo presentarán } \\
\text { el Directorde } \\
\text { Sistemas, la Gerente } \\
\text { de RRHH y el Gerente } \\
\text { de Recu ros físicos e } \\
\text { Inventarios }\end{array}$ & 100 \\
\hline & & $\begin{array}{l}\text { Crear un grupo de Whats } \\
\text { de gerentes y ases ores }\end{array}$ & \begin{tabular}{|c|} 
Formación del \\
grupo de \\
Whats App
\end{tabular} & No se realizó & 0 & Realizado & 100 \\
\hline & & $\begin{array}{c}\text { Añadir a la página Web } \\
\text { bs bgrosy acciones } \\
\text { generales de la empresa }\end{array}$ & $\begin{array}{l}\text { Página Web } \\
\text { actualizada }\end{array}$ & No se realizó & 0 & $\begin{array}{c}\text { Realizado. Se } \\
\text { establecerá } \\
\text { mensua mente. Que } \\
\text { pase la información } \\
\text { NKT }\end{array}$ & 100 \\
\hline & & $\begin{array}{c}\text { Crear un buzón de ideas } \\
\text { y su gerencias para } \\
\text { escribir mejoras, } \\
\text { felicitaciones y } \\
\text { comentarios para mejorar }\end{array}$ & $\begin{array}{c}\text { Creación del } \\
\text { buz ón y } \\
\text { revisarb cada } \\
\text { dia } 20 \text { de mes }\end{array}$ & No se realizó & 0 & $\begin{array}{l}\text { Se está viendo el } \\
\text { diseío }\end{array}$ & 60 \\
\hline & & $\begin{array}{c}\text { Rebomar las reuniones } \\
\text { con e Comité una vez por } \\
\text { quincena }\end{array}$ & \begin{tabular}{|c|} 
Un viemes \\
cada 15 dias \\
una hora. Que \\
cada quien \\
envie a la \\
Directora \\
Administrativa \\
sus temas dos \\
dias antes
\end{tabular} & $\begin{array}{c}\text { Se rebomaron las } \\
\text { reuniones con el } \\
\text { Comité cada dos } \\
\text { vienes }\end{array}$ & 100 & $\begin{array}{l}\text { Se mantienen las } \\
\text { reuniones cada dos } \\
\text { vernes }\end{array}$ & 100 \\
\hline
\end{tabular}

Figura 5: Resumen de las áreas de oportunidad trabajadas, estrategias y resultados

Los resultados muestran que existe diferencia significativa en los puntajes de la post prueba con respecto al diagnóstico inicial (pre prueba) en cuanto a Calidad de vida en la empresa ( $\mathrm{t}=-0,856, \mathrm{p}=0,000<0,05)$, permaneciendo en "De acuerdo"; Cuidado y preservación del medio ambiente $(t=-4,4$, $\mathrm{p}=0,005<0,05)$, pasando de "Más o menos de acuerdo" a "De acuerdo", y Relación con la comunidad ( $\mathrm{t}=-13,856, \mathrm{p}=0,001<0,05)$, pasando de "Más o menos de acuerdo" a "De Acuerdo". Aunque los resultados para "Trato ético hacia los involucrados con la empresa" fueron superiores para la post prueba, la diferencia no fue significativa ( $t=-2,298$, $p=0,07>0,05$ ), lo que indica que la percepción se mantiene sin cambio en "De acuerdo".

Estos resultados indican que los trabajadores están de acuerdo en que se propicia una adecuada calidad de vida, en que mejoraron los esfuerzos de la empresa para preservar el medio ambiente, en que se promueve un trato ético hacia el interior y exterior de la empresa y en que ésta colabora en el mejoramiento del medio ambiente, en comparación con antes. Esto se debe a que, debido a la intervención, se ha mejorado la comunicación interna, se han implementado estrategias para la preservación y mejoramiento del medio ambiente, está mejorando la relación con la comunidad y se han realizado esfuerzos para mejorar la calidad de vida laboral, especialmente al implementar las guardias y verificar las cargas de trabajo. Cabe mencionar que al final de esos cuatro meses, y en función de la mejoría del comportamiento socialmente responsable, la rotación del personal bajó a $5 \%$ en promedio y el porcentaje de alcance de metas, como indicador de la productividad, 


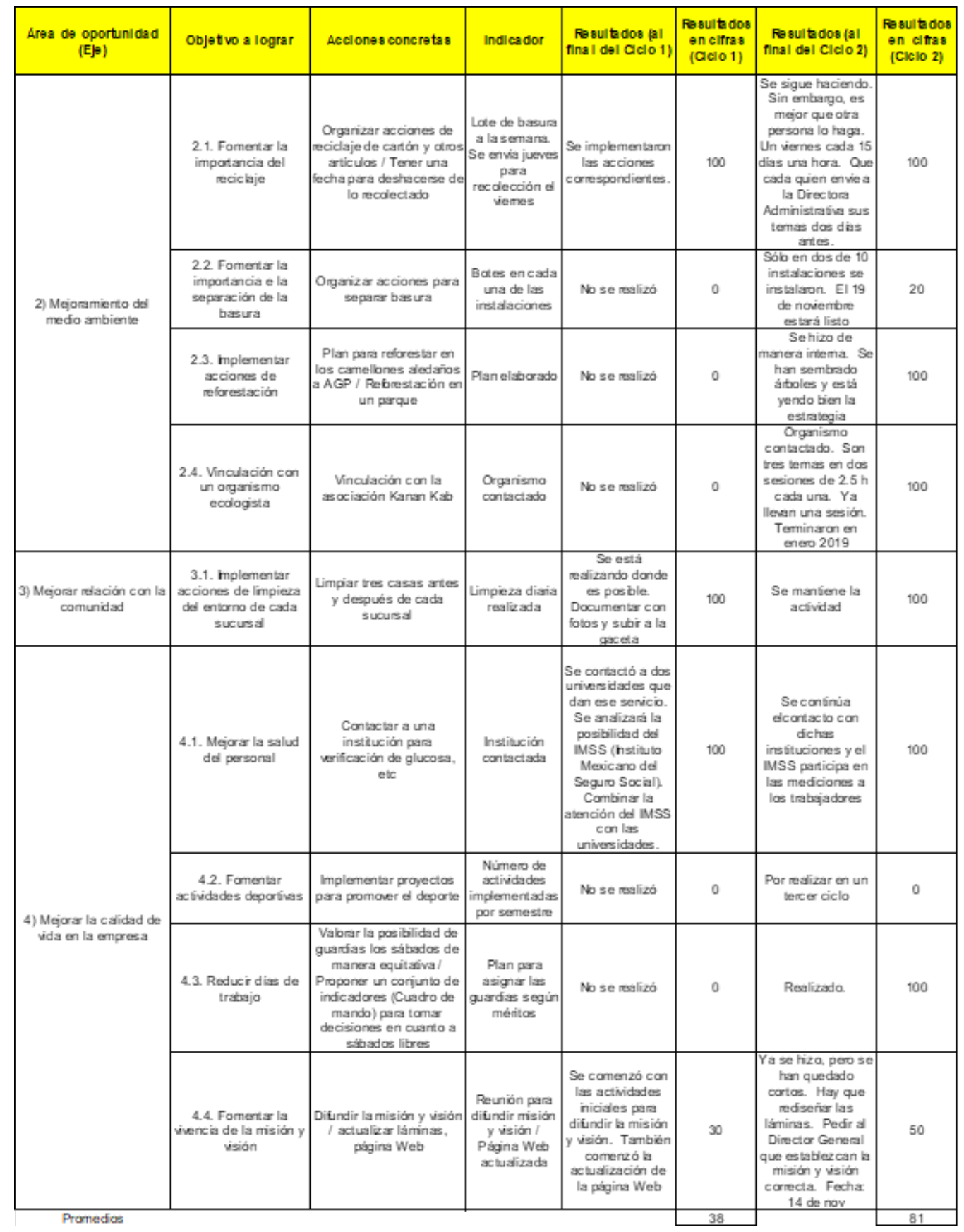

Figura 5: Resumen de las áreas de oportunidad trabajadas, estrategias y resultados (cont.)

pasó de $75 \%$ a $80 \%$ en promedio.

En la post prueba se registraron comentarios de los participantes en las preguntas abiertas al final del cuestionario, clasificadas por frecuencia de mención:
- Se tiene más conciencia en el cuidado del medio ambiente y en apoyar al trabajador en cuanto a esparcimiento

- Es interesante seguir esta metodología. Tenemos la capacidad y vamos a continuarla.

- Se toma en cuenta al personal en algunas 


\begin{tabular}{|c|c|c|c|}
\hline & Parte 1: Calidad de vida en la empresa & |Diagnóstico & Intervención \\
\hline 1 & La empresa apoya nuestro desarrollo & 3.9 & 4.0 \\
\hline 2 & La empresa nos capacita & 3.9 & 4.1 \\
\hline 3 & Los sueldos que paga la empresa son competitivos en el mercado laboral & 3.5 & 3.8 \\
\hline 4 & Contamos con prestaciones competitivas & 3.5 & 3.8 \\
\hline 5 & Puedo combinar mi trabajo con mi vida personal & 3.0 & 3.3 \\
\hline 6 & Las condiciones de trabajo son adecuadas & 3.8 & 4.0 \\
\hline 7 & Conozcolavisión de la empresa & 3.9 & 4.0 \\
\hline 8 & Conozcola misión de la empresa & 3.8 & 4.0 \\
\hline 9 & E liderazgo en la empresa es adecuado & 3.7 & 4.1 \\
\hline 10 & Se respeta nuestra dignidad como person $\approx$ & 4.0 & 4.3 \\
\hline & Promedio: Calidad de vida en la empresa & 3.7 & 3.9 \\
\hline
\end{tabular}

\begin{tabular}{|c|c|c|c|}
\hline & Parte 2: Cuidado y preservación del medio ambiente & Diagnóstico & Intervención \\
\hline 14. & La empresa se preocupa por el cuidado del medio ambiente & 3.7 & 3.7 \\
\hline 15 & La empresa fomenta en sus trabajadores el cuidado del medio ambiente & 3.3 & 3.8 \\
\hline 16. & La empresa participa en actividades que cuiden el medio ambiente & 2.8 & 4.0 \\
\hline 17 & Se capacita al personal para el cuidado del ambiente & 2.5 & 3.6 \\
\hline 18 & La empresa organiza actividades para el cuidado ambiental & 2.6 & 3.6 \\
\hline 19 & La empresa colabora con organismos o instituciones dedicadas al cuidado ambiental & 2.6 & 3.6 \\
\hline & Se respeta el entomo ecológico en sus procesos de operación. & 3.3 & 3.7 \\
\hline & Promedio: Quidado y preservación del medio ambiente & 3.0 & 3.7 \\
\hline
\end{tabular}

\begin{tabular}{|c|c|c|c|}
\hline & Parte 3 Trato ético hacia los involucrados con la empresa (stakeholders) & Diagnóstico & Intervención \\
\hline 21 & E trato de la empresa es justo hacia los que laboran en ella & 3.4 & 3.8 \\
\hline 22 & Subirde puesto en la empresa depende del esfuerzo de cada trabajador & 4.2 & 4.1 \\
\hline 23 & La comunicaxión en la empresa promueve un buen clima laboral & 3.6 & 4.0 \\
\hline 24. & Se toma en cuenta al personal en las decisiones de la empresa & 3,3 & 3.5 \\
\hline 25 & Se toma en cuenta a los extemos (accionistas, proveedores, clientes) en las decisiones de la empresa & 4.0 & 4.0 \\
\hline 26 & E trato de la empresa hacia los extemos (accionistas, proveedores, clientes) es justo & 3.6 & 4.2 \\
\hline & Promedio: Trato ético hacia los involucrados con la empresa [ & 3.7 & 3.9 \\
\hline
\end{tabular}

\begin{tabular}{|c|c|c|c|}
\hline & Parte 4: relación con la comunidad & Diagnóstico & Intervención \\
\hline 27 & La empresa ayuda a movimientos de caridad (filantropia) & 3.1 & 4.0 \\
\hline 28 & La empresa es importante para el desarrollo de la comunidad & 3.5 & 4.2 \\
\hline 29 & La empresa promueve hacia la comunidad una cultura de responsabilidad social & 3.4 & 4.1 \\
\hline $30 \mid$ & La empresa se desempeña con un código de ética & 3.3 & 4.2 \\
\hline & Promedio: Relación con la comunidad | & 3.3 & 4.1 \\
\hline
\end{tabular}

Figura 6: Resultados de pre prueba (diagnóstico) y post prueba

decisiones, pues antes no sucedía eso.

- Han mejorado las condiciones laborales, lo que motiva a participar más activamente en la empresa y ser más productivos.

Esto sugiere que además de implementar proyectos para promover el deporte (que quedó pendiente para un siguiente ciclo) y seguir trabajando en fomentar la vivencia de la misión y visión, la empresa debe trabajar en aspectos de equidad y revisar el reglamento por el asunto de los descuentos al personal. Sin embargo, deben analizarse periódicamente los logros ya conseguidos para mantenerlos o incrementarlos, es de- cir, pasar de "De acuerdo" a "Totalmente de acuerdo".

\section{Conclusiones}

A lo largo del proceso se notó el involucramiento del personal de la empresa. Se realizaban acciones de RSE, pero no eran suficientes y los trabajadores no estaban enterados de lo que se hacía al respecto. El personal de la empresa estaba de acuerdo con que la empresa tenía un comportamiento socialmente responsable. Sin embargo, luego de la intervención mejoró significativamente la calidad de vida del trabajador, la preservación del ambiente y la colaboración con la 
comunidad que, aunque siguen estando "De acuerdo", son mejores los puntajes. El trato ético no se incrementó sustancialmente, aunque también califica como "De acuerdo".

Las propuestas de cambio lograron mejoría significativa en tres de los cuatro ámbitos del CEMEFI, que se ubicaron en "De acuerdo" (ver escala de la Figura 2 y los resultados de la Figura 6), por lo que esta empresa es un ejemplo de que se puede ser socialmente responsable y seguir siendo rentable al mismo tiempo.

En cuanto a las causas por las que la empresa desarrolló un comportamiento socialmente responsable, el buen ambiente entre los miembros del Comité IAP contribuyó a consolidar su compromiso, además de que el liderazgo participativo ejercido por el Director General fue fundamental, lo que concuerda con Robbins y Judge (2013) y Goleman (2005) quienes sostienen que el estilo de liderazgo influye positivamente en el rendimiento del grupo. También fue evidente que el buen clima laboral mejoró en la empresa, y conforme fue mejorando, se vio a los trabajadores más entusiasmados para realizar sus labores. El mejoramiento del comportamiento socialmente responsable logró aumentar la productividad y reducir la rotación del personal, lo que para Barroso-Tanoira (2017) es consecuencia del mejoramiento de la calidad de vida de los trabajadores.

La investigación ha mostrado que, según el testimonio de los integrantes del Comité IAP, los trabajadores entienden más la importancia del comportamiento socialmente responsable. Los resultados arrojan que también han mejorado entre ellos la comunicación, el trabajo en equipo y el sentido de pertenencia a la empresa. Mediante el desarrollo de las actividades y la interacción con los representantes respectivos del Comité IAP, los participantes fueron sujetos e investigadores al mismo tiempo, como sugieren Bernal (2010) y Lois (s.f.). Eso, más el liderazgo del Director General, hicieron que se avanzara en las intervenciones y se lograran las metas que se alcanzaron. Demostraron su capacidad para seguir el proceso por sí mismos y lo han seguido haciendo.
Como recomendación, hay que continuar con los ciclos de intervención y, como indica Bernal (2010), lograr que la empresa sea gestora de su propio conocimiento a través de la crítica consciente y la reflexión constante. Y aunque una limitación fue que los resultados solo son válidos para esta empresa, sería interesante un estudio futuro en el que se replicara la metodología entre empresas de diferentes sectores, lo que podría llevar a un modelo que pueda aplicarse para fomentar el comportamiento socialmente responsable en empresas de diferente giro y tamaño. Esto podría fortalecer a las empresas para tomar decisiones en tiempos de crisis, como la pandemia de la COVID 19 que el mundo vive ahora, pues con la IAP las empresas aprenden a aprender, con lo que la adaptación al medio es más factible.

El autor de este trabajo agradece a la empresa AGP su autorización para reportar los resultados de este estudio y a la Oficina de Transferencia de la Universidad Anáhuac Mayab por las facilidades para realizar y reportar este trabajo.

\section{REFERENCIAS BIBLIOGRÁFICAS}

- AGP. (2018). Manual de operación de Refaccionaria AGP. Recuperado de https://www. refaccionariasagp.com/pages/conocenos - Álvarez-Gayou, J. (2003). Cómo hacer investigación cualitativa. Fundamentos y metodología. Colección Paidós Educador. México: Paidós Mexicana.

- Barroso-Tanoira, F. (septiembre-diciembre, 2008). La responsabilidad social empresarial. Un estudio en cuarenta empresas de la ciudad de Mérida, Yucatán. Contaduría y Administración, 226, 73-91.

- Barroso-Tanoira, F. (2017). Responsabilidad social empresarial: ¿estrategia para mejorar el desempeño laboral y aumentar utilidades, o deber ético de las empresas? Revista Universitaria RUTA (Universidad de La Serena, Chile), 19 (1), 1-25. doi http://dx.doi. org/10.15433/ruta.v18i1.869

- Bernal, C. (2010). Metodología de la investigación ( $2^{\mathrm{a}}$. ed.). México: Pearson Educación.

- Colmenares, A. M. (2012). Investigación- 
acción participativa: una metodología integradora del conocimiento y la acción. Voces y Silencios: Revista Latinoamericana de Educación, 3(1), 102-115. Recuperado de https://dialnet.unirioja.es/servlet/ articulo?codigo $=4054232$

De-Oliveira-Figueiredo, G. (2015). Investigación Acción Participativa: una alternativa para la epistemología social en Latinoamérica. Revista de Investigación. 39(86): 271-290. - Elliott, J. (2000). El cambio educativo desde la investigación-acción ( $4^{\mathrm{a}}$ ed.). Madrid: Morata.

- Fernández-García, R. (2018). La responsabilidad social corporativa como modelo de gestión empresarial. Barcelona, España: Bosch. 201 Pp.

- Goleman, D. (2005). Liderazgo que obtiene resultados. Clásicos HBR. Harvard Business School Publishing Corporation.

- Hernández-Sampieri, R. y Mendoza, C. (2018). Metodología de la investigación. Las rutas cuantitativa, cualitativa y mixta. México: McGraw Hill Educación. 714 Pp.

- Lois, I. (s.f.). La investigación-acción (I+A) y la investigación acción participativa (IAP): un recorrido posible entre el conocimiento y la praxis. Recuperado de http://www. sociales.uba.ar/wp-content/blogs.dir/219/ files/2017/08/15-Lois.pdf

- Pérez-Espinoza, M. J., Espinoza-Carrión, C. y Peralta, B. (2016). La responsabilidad social empresarial y su enfoque ambiental: una visión sostenible a futuro. Universidad y sociedad. 8(3): 169-178. Disponible en: http://scielo.sld.cu/pdf/rus/v8n3/rus23316.pdf. Fecha de consulta: 1 de noviembre de 2020.

Porto, N. y Castromán, J. (septiembre-diciembre, 2006). Responsabilidad social: un análisis de la situación actual en México y España. Contaduría y Administración, FCAUNAM, 220, 67-87.

- Ramos, H. (primer semestre, 2006). Ética y responsabilidad social. Reflexiones y perspectiva sistémica. The Anáhuac Journal, 6(1), 56-71.

- Robbins, S. P. y Judge, T. A. (2013). Comportamiento organizacional (15a. ed.). México: Pearson.

- Rojas-de-la-Puente, E. E., RimarachínChávez, F. L., Oblitas-Cruz, J. y Castro-Silupu, W. (2019). Control de proyectos de responsa- bilidad social. Un estudio en empresas mineras. Revista Venezolana de Gerencia. 24(87): 684-700.

- Secretaría de Economía (30 de junio de 2009). Acuerdo por el que se establece la estratificación de las micro, pequeñas y medianas empresas. Diario Oficial de la Federación. Recuperado de https://www.economia. gob.mx/files/marco_normativo/A539.pdf

- Sirvent, M. (2008). Educación Popular y Universidad Pública. Ensayo para una historia que aún espera ser escrita. Revista del IICE, (3), 51-68. Recuperado de http://revistascientificas.filo.uba.ar/index.php/iice/article/ view/365/350 